\title{
Impact of Integrating Pet-Ct in Radiotherapy Planning of Non-small Cell Carcinoma Lung: Dosimetric and Radiobiological Comparison
}

\author{
Deepak Koppaka1, Rakesh Kapoor ${ }^{1}$, Amit Bahl' ${ }^{1}$, Anshuma Bansal', BR Mittal ${ }^{2}$ and Navneet Singh ${ }^{3}$
}

${ }^{1}$ Department of Radiotherapy, PGIMER, Chandigarh, India

${ }^{2}$ Department of Nuclear Medicine, PGIMER, Chandigarh, India

${ }^{3}$ Department of Pulmonary Medicine, PGIMER, Chandigarh, India

\begin{abstract}
The integration of PET and CT scans allows the simultaneous use of biologic and anatomic imaging data for better delineating tumor and sparing normal critical structures. The aim of this study is to assess the impact on target volume delineation using CT vs. PETCT based plans, and to determine radiobiological effect on tumor and normal tissue by comparing Tumor control probability (TCP) \& normal tissue complication probability (NTCP) of CT \& PET-CT based plans. 15 patients of inoperable NSCLC planned for radical radiotherapy, underwent a planning CT scan of the thorax. Target volumes were contoured. Later PETCT images with the auto contoured MTV (Metabolic target volume) images, were fused with the planning CT images. Margins were similarly given to PETMTV for the generation of PET CTV and PET PTV. 3 Dimensional conformal radiotherapy (3DCRT) plans were made on the planning $C T$ images and the planning PETCT images, and were compared dosimetrically and radio biologically. The study shows that the use of PETCT resulted in significant decrease in PETCT generated MTV versus GTV delineated on CT. Also, there was statistical significant decrease in the volume of the normal lung irradiated in terms of V40 and the MLD. TCP for PETCT based plans was significantly higher than that with CT based plans. Also, PETCT based planning resulted in a statistically significant decrease in the NTCP for lung and spinal cord. Therefore, the study concludes that incorporation of PETCT into radiotherapy planning of NSCLC is technically feasible and dosimetrically appealing strategy for patient treatment.
\end{abstract}

Keywords: Non-small cell lung cancer; PET: Positron emission tomography; CT: Computed tomography; Radiotherapy planning

\section{Introduction}

Radiotherapy nowadays is playing a significant role in the management of Non-small cell lung cancer (NSCLC), both in early stages as stereotactic body radiotherapy, as well as in late stages, where, radiotherapy with chemotherapy is the treatment of choice. When delivered by conventional techniques, 3 - and 5 -year disease-specific survivals of $39 \pm 10 \%$ and $25 \pm 9 \%$, and overall survival of $34 \pm 9 \%$ and $21 \pm 8 \%$, respectively have been reported in literature [1]. Poorer survival rates with conventional radiotherapy in comparison to surgery can be contributed to suboptimal staging of patients, insufficiently high radiation doses, 'geographical misses' owing to tumor mobility, greater normal lung tissue toxicity, as well as toxicity to organs such as heart, spinal cord, esophagus, which are in the vicinity of tumor tissues.

Keeping these issues in mind, radiation oncologists are continually seeking various new methods to target radiotherapy more precisely. 3 Dimensional conformal radiation therapy (3 DCRT) technique has largely been successful in overcoming these difficulties. Computed tomography (CT) scan has been the main source of anatomic imaging for tumor volume delineation in 3DCRT. However it has limited resolution for soft tissue and tumor delineation, resulting in missing the tumor volume such as mediastinal lymph nodes, or over-treating the tumor volume due to atelectasis, As a consequence high doses of irradiation could not be delivered to the tumor without risking normal lung tissue. This is a great disadvantage, since local progression has been shown to be the leading cause of death in irradiated NSCLC patients.

Fluoro-2-deoxy-D-glucose (FDG)-positron emission tomography (PET) allows the preferential accumulation of radiotracers in the neoplastic cells, differentiating them from the benign tissue. The integration of PET and CT scans in NSCLC allows the simultaneous use of biologic and anatomic imaging data which definitely has an advantage over CT alone, as PET CT can better detect lymph node metastases that are missed on a CT scan, and also it can better differentiate the tumor volume from post obstructive atelectasis, thereby decreasing the normal lung irradiation [2,3]. In addition use of PET image data into target volume delineation has consistently shown a reduction in inter-observer variation due to the automated segmentation which is possible in PET CT, as compared to CT-based delineation where target volume delineation is mainly carried out manually. PET-CT thus allows for advanced radiotherapy delivery, however care must be taken to avoid a technical limitations [4].

This study presents the impact of FDG-PET CT integration on tumor delineation and its coverage, and consequently on the doses to critical organs such as the esophagus and spinal cord for a given treatment technique. Also the feasibility of incorporating PET-CT data into treatment planning in the present setup has been evaluated. The aim is to assess the impact on target volume delineation using CT vs. PETCT based plans, and to determine the radiobiological effect on tumor and normal tissue by comparing Tumor control probability (TCP) and normal tissue complication probability (NTCP) of CT \& PET-CT based plans.

*Corresponding author: Deepak Koppaka, Department of Radiotherapy, Nehru Hospital, PGIMER, Sector 12, Chandigarh, Tel: 09592096223; E-mail: drdeepak.koppaka@gmail.com

Received June 26, 2015; Accepted July 13, 2015; Published July 20, 2015

Citation: Koppaka D, Kapoor R, Bahl A, Bansal A, Mittal BR, et al. (2015) Impact of Integrating Pet-Ct in Radiotherapy Planning of Non-small Cell Carcinoma Lung: Dosimetric and Radiobiological Comparison. J Integr Oncol 4: 139. doi:10.4172/2329-6771.1000139

Copyright: @ 2015 Koppaka D, et al. This is an open-access article distributed under the terms of the Creative Commons Attribution License, which permits unrestricted use, distribution, and reproduction in any medium, provided the original author and source are credited. 
Citation: Koppaka D, Kapoor R, Bahl A, Bansal A, Mittal BR, et al. (2015) Impact of Integrating Pet-Ct in Radiotherapy Planning of Non-small Cell Carcinoma Lung: Dosimetric and Radiobiological Comparison. J Integr Oncol 4: 139. doi:10.4172/2329-6771.1000139

Page 2 of 6

\section{Materials and Methods}

The study population included 15 patients of inoperable NSCLC attending radiotherapy outpatient department, PGIMER, Chandigarh. The patients were planned to undergo treatment with 3D-CRT technique using PET-CT based plans. The inclusion criteria for taking the patients into study was, previously untreated histological proven cases of NSCLC, either medically or surgically inoperable (Stage I - IIIB), or those who had completed chemotherapy (if planned) 4 weeks back. All patients had Karnofsky performance status (KPS) $\geq$ 70 and adequate hematological and biochemical parameters. Patients who were less than 18 years of age, pregnant, had prior malignancy of any other site, stage IV disease and patients diagnosed with small cell carcinoma and atypical carcinoids were excluded from the study. Patients were evaluated by doing a thorough clinical examination followed by the baseline investigations like hemogram, liver Function Tests, kidney Function Test, chest X-ray, bronchoscopy, CECT chest and bone scan.

\section{Methodology}

All the patients initially underwent a planning CT scan of the thorax after an informed consent. They were laid in supine position with both arms raised holding the T- bar and breathing freely. They were counseled to maintain normal breathing pattern and no thermoplastics were used for immobilization. For tumor localization, a planning CT scan was taken in each patient using CT simulator machine GE Light Speed VFX with $2.5 \mathrm{~mm}$ slice thickness. After intravenous contrast, patients were scanned from level of neck to the level of upper abdomen, including both adrenals.

Target volume delineation: Target volumes were contoured according to International commission on radiation units and measurements (ICRU) 50 recommendations on the planning CT DICOM images. This contouring was done prior to planning PETCT to avoid bias in contouring. Gross tumor volume (GTV) consisted of the macroscopic tumor and included metastatic lymphadenopathy greater than $1 \mathrm{~cm}$ in size. Clinical target volume (CTV) is the expansion of GTV in order to account for the spread of subclinical disease. It was taken as GTV plus a $6 \mathrm{~mm}$ margin for squamous cell carcinoma and 8 $\mathrm{mm}$ margin for adenocarcinoma. For lymph nodes, margin of $8 \mathrm{~mm}$ was taken. Planning target volume (PTV) was calculated from CTV with margin of $8-12 \mathrm{~mm}$.

Later a planning 18 F-FDG-PET-CT scan was taken in the department of Nuclear medicine, using a PET scanner Discovery STE16LS (GE healthcare). Patients were kept fasting for at least 4 hours and blood glucose level $<150 \mathrm{mg} / \mathrm{dl}$ were ensured. Immediately prior to PETCT acquisition, all patients were asked to empty their bladders. Scanning was initiated 45 minutes after oral and intravenous administration of 10-15 mCi of FDG. The following requirements were ensured for PET-CT planning: Flat table top, matching of table axis for both PET-CT \& CT, selection of same field of vision by both CT \& PET-CT, selection of patient positioning (similar to planning CT), transfer of data from PET-CT to treatment planning system (TPS), registration of images using same co-ordinate system i.e. using bony contour of the patient.

After image acquisition on PETCT, Metabolic Tumor Volume (MTV) was defined. MTV is generated using a fixed tumor background ratio 1.5 times of the mean activity of aorta. Any node showing enhancement on PET-CT was contoured irrespective of the size of the lymph-node.
The PETCT images with the auto contoured MTV images were then fused with the planning CT images using rigid bony registration.

Margins were similarly given to PET MTV for the generation of PET CTV and PET PTV as per the ICRU 50 recommendations.

Organ at risk (OAR) contouring: OARs contoured were spinal cord, esophagus, heart and remaining lung parenchyma

Treatment planning: Treatment plans were initially made on the planning CT images using an antero-posterior and postero-anterior (AP-PA) fields in most cases keeping in mind the tolerance of various organs, mainly the normal lungs and spinal cord. Similar treatment plans were then made on the planning PETCT images. CT and PETCT based dose calculation were done using 3D-TPS.

Required dose homogeneity within PTV was limited within 95\% to $107 \%$ of primary dose. Dose less than $95 \%$ was accepted for $1 \%$ of PTV. The patients were treated on the PET-CT based plans with a dose of 66 Gy delivered in 33 fractions in 6.5weeks after an evaluation of OAR dose constraints (Table 1).

These 3D-CRT plans generated using the PET-CT images were then compared with the 3D-CRT plans generated using CT images both dosimetrically from data obtained from Dose volume histograms (DVH) and radiobiologically from mathematical models like TCP and NTCP.

Statistical analysis: Statistical analysis of data was done using Statistical Package for Social Sciences (SPSS) version 19. Descriptive statistics analyzed using mean, median, and percentage, were used to measure and compare doses for various organs at risk and for target volumes. TCP and NTCP were calculated by using POISSEN model and LKB model respectively [5-8]. To compare TCP, NTCP \& volumes contoured by CT \& PET-CT, Wilcoxon signed rank test was used. All tests were two tailed and $p$ value $<0.05$ was taken as significant.

\section{Results}

Table 2 shows the characteristics of the patients included in the study.

\section{Target volumes}

GTV vs. MTV: Table 3 compares GTV delineated using CT scans

\begin{tabular}{|c|l|}
\hline Organ at risk & Constraint \\
\hline Lung & \begin{tabular}{|c|} 
V20 (volume of lung receiving $20 \mathrm{~Gy})<30-35 \%$ \\
Mean Lung Dose (MLD) $<20-23 \mathrm{~Gy}$
\end{tabular} \\
& $\begin{array}{l}\text { V10 (volume of lung receiving } 10 \mathrm{~Gy})<45 \% \\
\text { Limiting dose to central airway dose }<80 \mathrm{~Gy}\end{array}$ \\
\hline Heart & V40 (volume of heart receiving $40 \mathrm{~Gy})<50 \%$ \\
\hline Esophagus & V55 (volume of esophagus receiving $55 \mathrm{~Gy})<50 \%$ \\
\hline Spinal cord & Dmax (maximum dose at any point) $<45 \mathrm{~Gy}$. \\
\hline \multicolumn{2}{|c|}{ Table 1: Dose volume constraints for organ at risk. }
\end{tabular}

\begin{tabular}{|c|c|}
\hline Mean age & $\mathbf{5 9 . 2}$ yrs $\mathbf{( 4 5}$ yrs $\mathbf{6 5} \mathbf{~ y r s})$ \\
\hline Male : Female & $13: 2$ \\
\hline Stage IIB & 3 patients \\
IIIA & 5 patients \\
IIIB & 7 patients \\
\hline Histology : \\
Adenocarcinoma & 5 patients \\
\hline Squamous cell carcinoma & 10 patients \\
\hline Chemotherapy: & \\
\hline Induction chemotherapy & 7 patients \\
Concurrent chemotherapy & 8 patients \\
\hline
\end{tabular}

Table 2: Patient characteristics. 
Citation: Koppaka D, Kapoor R, Bahl A, Bansal A, Mittal BR, et al. (2015) Impact of Integrating Pet-Ct in Radiotherapy Planning of Non-small Cell Carcinoma Lung: Dosimetric and Radiobiological Comparison. J Integr Oncol 4: 139. doi:10.4172/2329-6771.1000139

Page 3 of 6

and MTV delineated using PETCT scans. It shows a decrease in the magnitude of the GTV on using PETCT data in 14 patients, which is statistically significant $(\mathrm{p}=0.001)(\mathrm{z}=-3.35)$. The decrease in volume per patient varied from $3.68 \%$ to $89.23 \%$. Tumor delineation using PETCT resulted on an average $36.18 \%$ lesser volume than a CT scan (Table 4 ).

PETCT NODE VS CT NODE: Unlike the primary tumor, PETCT leads to identification of greater number of lymph nodes than on a CT scan, resulting in greater MTV nodal volumes than GTV nodal volumes, and the difference is statistically significant $(\mathrm{p}=0.05)(\mathrm{z}=-$ 1.93) (Table 3). Using PETCT for nodal delineation, resulted in about $62.60 \%$ greater volumes than a CT scan (Table 4 ).

CTV and PTV: Except for four patients, CTV and PTV delineated using PETCT were significantly less compared to those delineated using CT scan (Table 5). PETCT resulted in a decrease in CTV and PTV by about $22.48 \%$ and $16.69 \%$ respectively on an average when compared with CT volumes (Table 6). In patients where the PET PTV volumes were greater than CT PTV volumes, the difference ranged from $1.55 \%$ to $20.61 \%$.

\section{Organ at risk doses}

Spinal cord dose : The mean spinal cord Dmax was less in PETCT based plans (43.94 Gy) compared to CT based plans ( $47.46 \mathrm{~Gy}$ ) by $6.46 \%$ and this decrease in dose reached statistical significance $(\mathrm{p}=0.015)(\mathrm{z}=-2.56)$. The maximum decrease in spinal cord dose was as high as $38.45 \%$ while the maximum increase in spinal cord dose was $31.55 \%$ (Table 7 ).

Esophagus dose: The impact of incorporating PETCT in planning

\begin{tabular}{|c|c|c|c|c|}
\hline MEAN & $\begin{array}{c}\text { PET MTV } \\
\text { (in cc) }\end{array}$ & $\begin{array}{c}\text { CT GTV } \\
\text { (in cc) }\end{array}$ & $\begin{array}{c}\text { PET MTV NODE } \\
\text { (in cc) }\end{array}$ & $\begin{array}{c}\text { CT GTV NODE } \\
\text { (in cc) }\end{array}$ \\
\hline MEDIAN & 102.69 & 157.30 & 26.27 & 15.96 \\
\hline $\mathbf{p}$ & 102.93 & 145.81 & 8.9 & 5.30 \\
$\mathbf{z}$ & $0.001(\mathrm{MTV} / \mathrm{GTV})$ & & $\begin{array}{c}0.05(\mathrm{MTV} / \mathrm{GTV} \\
\text { node) } \\
-1.93\end{array}$ & \\
\hline
\end{tabular}

Table 3: Comparison of PETCT MTV and CT GTV showing a decrease in PET MTV; and PET MTV node and CT GTV node showing an increase in PET MTV node volume. Abbreviations MTV Metabolic tumor volume, GTV Gross tumor volume. PET Positron Emission Tomography CT Computer Tomography.

\begin{tabular}{|l|c|c|}
\hline & $\begin{array}{c}\text { Number of } \\
\text { patients (\%) }\end{array}$ & Range (\%) \\
\hline GTV > MTV & $14(94.4 \%)$ & $3.68 \%-89.23 \%$ \\
\hline GTV < MTV & $1(6.6 \%)$ & $4.35 \%$ \\
\hline Net decrease in PET MTV vs CT GTV $=36.18 \%$ & 4 & $23.45 \%-65.95 \%$ \\
\hline GTV node > MTV node & 11 & $40.74 \%-260.09 \%$ \\
\hline GTV node < MTV node & \multicolumn{2}{|l}{} \\
\hline Net increase in nodal delineation by PETCT vs CT = $62.60 \%$ &
\end{tabular}

Table 4: Net change in target volumes (MTV/GTV) PETCT vs CT. Abbreviations MTV Metabolic tumor volume, GTV Gross tumor volume. PET Positron Emission Tomography CT Computer Tomography.

\begin{tabular}{|c|c|c|c|c|}
\hline & $\begin{array}{l}\text { PET CTV } \\
\text { (in cc) }\end{array}$ & CT CTV & $\begin{array}{l}\text { PET PTV } \\
\text { (in cc) }\end{array}$ & $\begin{array}{c}\text { CT PTV } \\
\text { (in cc) }\end{array}$ \\
\hline MEAN & 334.26 & 432.60 & 800.47 & 949.44 \\
\hline MEDIAN & 309.53 & 365.90 & 737 & 837 \\
\hline$p$ & 0.006 (CTV) & & 0.009 (PTV) & \\
\hline$z$ & 2.72 & & 2.61 & \\
\hline
\end{tabular}

Table 5: Comparison of PETCT CTV and CT CTV; and PETCT PTV and CT PTV Abbreviations: CTV Clinical Target Volume, PTV Planning Target Volume, PET Positron Emission Tomography, CT Computer Tomography.

\begin{tabular}{|l|c|c|}
\hline & Number of patients (\%) & Range (\%) \\
\hline CT CTV < PET CTV & 4 & $0.75 \%-40.09 \%$ \\
\hline CT CTV > PET CTV & 11 & $2.87 \%-67.70 \%$ \\
\hline Net decrease in CTV on PETCT vs CT $=22.48 \%$ & \multicolumn{2}{|l|}{} \\
\hline CT PTV < PET PTV & 4 & $1.55 \%-20.61 \%$ \\
\hline CT PTV > PET PTV & 11 & $12.56 \%-50.44 \%$ \\
\hline
\end{tabular}

Net decrease in PTV on PETCT vs CT $=16.69 \%$

Table 6: Net change in target volumes (CTV and PTV) PETCT vs CT Abbreviations : CTV Clinical Target Volume, PTV Planning Target Volume, PET Positron Emission Tomography , CT Computer Tomography.

\begin{tabular}{|l|c|c|} 
& Number of patients (\%) & Range (dmax) \\
\hline CT SC dmax < PET SC dmax & 2 & $1.57 \%-31.55 \%$ \\
\hline CT SC dmax > PET SC dmax & 13 & $0.68 \%-38.45 \%$
\end{tabular}

Net Decrease in Dose of Spinal Dmax $=6.46 \%$

Table 7: Net change in spinal cord dose PETCT vs CT. Abbreviations : SC Spina cord, dmax Dose maximum , PET Positron Emission Tomography , CT Computer Tomography.

\begin{tabular}{|c|c|c|c|c|c|c|c|c|}
\hline & CT & PETCT & $\begin{array}{c}\mathbf{p} \\
\text { Value }\end{array}$ & $\begin{array}{c}\Delta \mathbf{n} \\
\text { Increase }\end{array}$ & Range & $\begin{array}{c}\Delta \mathbf{n} \\
\text { Decrease }\end{array}$ & Range & $\begin{array}{c}\text { Net } \\
\text { decrease }\end{array}$ \\
\hline Dmax & 62.0 & 59.24 & 0.006 & 2 & $\begin{array}{c}0.34 \%- \\
2.52 \%\end{array}$ & 13 & $\begin{array}{c}0.18 \%- \\
39.12 \%\end{array}$ & $4.8 \%$ \\
\hline V50 & 16.71 & 11.56 & 0.05 & 3 & $\begin{array}{c}0.15 \%- \\
303.97 \%\end{array}$ & 9 & $\begin{array}{c}5.38 \%- \\
86.28 \%\end{array}$ & $12.18 \%$ \\
\hline V55 & 12.25 & 7.82 & 0.034 & 3 & $\begin{array}{c}7.81 \%- \\
333.6 \%\end{array}$ & 9 & $\begin{array}{c}12.96 \%- \\
99.56 \%\end{array}$ & $14.49 \%$ \\
\hline
\end{tabular}

V50, V55 (Volume of esophagus receiving 50 Gy, 55 Gy respectively) $\Delta \mathrm{n}$ (Number of patients)

Table 8: Esophagus dose.

\begin{tabular}{|c|c|c|c|c|c|c|c|c|}
\hline & CT & PETCT & $\begin{array}{c}\mathbf{p} \\
\text { Value }\end{array}$ & $\begin{array}{c}\Delta \mathbf{n} \\
\text { Increase }\end{array}$ & Range & $\begin{array}{c}\Delta \mathbf{n} \\
\text { Decrease }\end{array}$ & Range & $\begin{array}{c}\text { Net } \\
\text { decrease }\end{array}$ \\
\hline Mean & 20.24 & 18.73 & 0.078 & 3 & $\begin{array}{c}0.89 \%- \\
108.6 \%\end{array}$ & 12 & $\begin{array}{c}1.29 \%- \\
49.58 \%\end{array}$ & $8.09 \%$ \\
\hline V40 & 25.15 & 23.62 & 0.30 & 4 & $\begin{array}{c}3.19 \%- \\
166.07 \%\end{array}$ & 10 & $\begin{array}{c}3.5 \%- \\
70.53 \%\end{array}$ & $1.98 \%$ \\
\hline
\end{tabular}

Abbreviations: PET Positron Emission Tomography , CT Computer Tomography V40 (Volume of heart receiving $40 \mathrm{~Gy}$ )

$\Delta \mathrm{n}$ (Number of patients)

Table 9: Heart dose.

has resulted a decrease in esophagus Dmax by $4.8 \%$, and D 55 by $14.49 \%$ when compared to CT based planning (Table 8).

Heart dose: Though use of PETCT resulted in less radiation dose to the heart, no statistically significant change was observed. About $8.09 \%$ less mean dose was received by heart when PETCT was used (Table 9).

Normal lung dose: PETCT resulted in a decrease in various lung doses but decrease in V40 and MLD doses only, reached statistical significance. Use of PETCT based planning resulted in a decrease of V20,V10,V5 by $5.39 \%, 4.70 \%, 3.95 \%$ in comparison to CT planning (Table 10).

\section{Comparison of NTCP and TCP}

NTCP calculated by LKB models (2) showed a statistically significant decrease in the NTCP for lung and spinal cord. Though PETCT based plans resulted in decrease in NTCP for esophagus but it did not reach statistical significance (Table 11).

As calculated by Poisson's statistics TCP to the GTV was increased in PETCT based plans which reached statistical significance. 
Citation: Koppaka D, Kapoor R, Bahl A, Bansal A, Mittal BR, et al. (2015) Impact of Integrating Pet-Ct in Radiotherapy Planning of Non-small Cell Carcinoma Lung: Dosimetric and Radiobiological Comparison. J Integr Oncol 4: 139. doi:10.4172/2329-6771.1000139

Page 4 of 6

\begin{tabular}{|c|c|c|c|c|c|c|c|c|}
\hline & CT & PETCT & $\begin{array}{c}\mathbf{p} \\
\text { Value }\end{array}$ & $\begin{array}{c}\mathbf{\Delta n} \\
\text { Increase }\end{array}$ & Range & $\begin{array}{c}\mathbf{\Delta n} \\
\text { Decrease }\end{array}$ & Range & $\begin{array}{c}\text { Net } \\
\text { Change }\end{array}$ \\
\hline V40 & 26.72 & 24.56 & 0.027 & 4 & $\begin{array}{c}1.10 \%- \\
2.79 \%\end{array}$ & 11 & $\begin{array}{l}5.88 \%- \\
45.23 \%\end{array}$ & $10.61 \%$ \\
\hline V20 & 36.59 & 35.12 & 0.11 & 5 & $\begin{array}{c}0.67 \%- \\
14.30 \%\end{array}$ & 10 & $\begin{array}{c}1.03 \%- \\
22.65 \%\end{array}$ & $5.39 \%$ \\
\hline V10 & 41.77 & 40.19 & 0.19 & 6 & $\begin{array}{l}0.09 \%- \\
14.44 \%\end{array}$ & 9 & $\begin{array}{c}4.43 \%- \\
21.48 \%\end{array}$ & $4.70 \%$ \\
\hline V5 & 46.13 & 44.71 & 0.21 & 6 & $\begin{array}{c}0.17 \%- \\
11.76 \%\end{array}$ & 9 & $\begin{array}{c}4.52 \%- \\
19.02 \%\end{array}$ & $3.95 \%$ \\
\hline MLD & 20.58 & 19.23 & 0.017 & 3 & $\begin{array}{l}1.35 \%- \\
14.73 \%\end{array}$ & 12 & $\begin{array}{c}3.34 \%- \\
29.07 \%\end{array}$ & $8.28 \%$ \\
\hline
\end{tabular}

V 40, V20, V10, V5 (volume of lung receiving 40 Gy, 20 Gy, 10 Gy, 5 Gy respectively)

MLD (Mean Lung Dose)

$\Delta \mathrm{n}$ (Number of patients)

Table 10: Lung dose.

\begin{tabular}{|c|c|c|c|}
\hline \multicolumn{5}{|c|}{ CT(\%) } & PETCT(\%) & p value \\
\hline Lung & $7.62(2.39-16.27)$ & $7.03(1.27-22.40)$ & 0.027 \\
\hline Esophagus & $9.28(1.70-17.83)$ & $7.53(1.52-15.11)$ & 0.069 \\
\hline Spine & $0.80(0.047-2.59)$ & $0.46(0.005-1.004)$ & 0.023 \\
\hline \multicolumn{3}{|c|}{ TCP } \\
\hline GTV & $\begin{array}{c}85.95(82.49- \\
89.92)\end{array}$ & $\begin{array}{c}86.39(83.91- \\
90.23)\end{array}$ & 0.020 \\
\hline
\end{tabular}

TCP Tumor Control Probability, NTCP Normal Tissue Complication Probability, PET Positron Emission Tomography , CT Computer Tomography

Table 11: NTCP and TCP.

\section{Discussion}

In the treatment of NSCLC, optimal dose delivery is a prerequisite for successful local tumor control. This should be achieved by avoiding geographic misses and unnecessary exposure of non-target tissue to radiation. CT-assisted volume definition remains the standard for external beam radiation therapy with curative intent. But with the incorporation of metabolic imaging in radiation treatment planning it has raised hopes for further improvement in target delineation [9]. For radiation therapy planning, the inclusion of FDG imaging produces significant changes in understanding the disease extent, location, size, and shape for patients with NSCLC [4]. In this study, we systematically evaluated the degree to which CT and PET define the volume of interest for treatment planning in NSCLC and whether the combination of PET and CT imaging provide data that is superior to CT imaging data alone for the purposes of radiation treatment (RT) planning.

In this study we have seen that using PETCT for delineation of target resulted on an average $26.5 \%$ decrease in total GTV/MTV. Greatest decrease in GTV was as high as $83.5 \%$ in a single patient. But in three patients use of PETCT resulted in greater lymph node delineation which resulted in greater total GTV volume than that on using a CT scan. In our analysis for better understanding the impact of PETCT on GTV delineation, GTV/MTV were contoured separately for the primary and for lymph nodes. It was seen that MTV of the primary tumor delineated using PETCT was significantly less than the GTV delineated using CT data. Using PET resulted in the decrease of the primary GTV by $54.61 \mathrm{cc}$ on an average. The magnitude of the average decrease in the tumor volume was about $36.19 \%$ when compared with CT GTV volumes.

Due to the inherent high sensitivity of PETCT in lymph nodal detection in NSCLC it is seen that the MTV nodal volumes delineated using PETCT were significantly higher than those delineated using CT images [10]. In eleven cases greater number of lymph nodes was delineated using PETCT. The magnitude of increase in lymph node volume by using PETCT is about $62.60 \%$ and was statistically significant. The greatest increase reported for any single patient was $260.09 \%$.

Other studies have also shown similar impact of PETCT on GTV delineation. For example, Bradley et al in their study have shown that in $58 \%$ of total cases PETCT resulted in an altered volume and in these $71 \%$ cases changes occurred from an altered nodal volume [11]. Similar study by De Ruysscher et al. found change in $67 \%$ of RT planning volumes due to changes in lymph node inclusion or exclusion [12]. Study by Deniaud-Alexandre et al have shown that total volume changes related to lymph nodal volume change in $19 \%$ of cases while in $81 \%$ of cases it occurred due to change in the primary lung volume which was either due to atelectasis or due to inter observer variability in target delineation [13]. Even in this present study only in 3 cases the total GTV change was due to change in lymph nodal volume while in the remaining 12 cases the change in GTV was due to change in the primary tumor volume. Ashamalla et al. has reported changes in only patients experiencing changes in GTV of $25 \%$ or more and it was seen $53 \%$ patients had change in GTV volumes [14]. In this study eleven patients had changes in the GTV volume of greater than $25 \%$.

Results regarding the impact of PETCT on CTV/PTV are mixed. Erdi et al has shown that use of PETCT resulted in an increase in PTV in about $20 \%$ cases [15]. Similar results were seen in a study by Brianzoni et al where CTV volume changes were seen in $44 \%$ cases of which only $20 \%$ patients had decrease in PTV while about $24 \%$ had increase in PTV because of increased lymph node delineation and better GV delineation [16]. Deniaud-Alexandre et al reported a decrease in PTV by $25 \%$ in $11 \%$ cases while increase in PTV by more than $25 \%$ in 12 patients [13]. While in our study only 6 patients had decrease in PTV of more than 25\% and none had an increase in PTV of more than $25 \%$. In this study we have seen that the main factor resulting in decrease CTV/PTV was the change in primary lung tumor volume. The magnitude of this change in the primary tumor volume was higher than the magnitude of change in the lymph nodal volume. Similar results were seen in a study by Schmucking et al. where about $92 \%$ patients experienced a decrease in tumor volume with the use of PETCT [17].

The incorporation of PETCT data into radiotherapy planning has resulted in a significant less dose to the normal lung than that seen with CT based treatment planning, as seen in this study. PETCT resulted in a decrease of V40 and MLD by $10.61 \%$ and $8.28 \%$ respectively. This decrease in V40 and MLD were statistically significant but the decrease in V20, V10, V5 were about 5.39\%, 4.70\%, 3.95\% respectively, which were not statistically significant.

Similar results were seen in a study by Mah et al, where the V20 values were not statistically significant when CT and PETCT based plans were compared [18]. Bradley et al. reported an increase in the V20 in $62 \%$ patients with the magnitude of increase upto $7.3 \%$ on an average [11]. This study also showed an increase in the MLD with the use of PETCT. This can be attributed to the increased target volume delieated by PETCT in this study. Other studies by De Ruysscher et al. and Van de Wel et al. reported a statistically significant decrease in the V20 and MLD doses [12,19].

Studies have shown that when GTV is decreased in patients with atelectasis, doses to normal tissues like esophagus also decrease thereby decreasing the probability of esophagitis. In this study we have seen that decrease in the tumor volume using PETCT resulted in a 
Citation: Koppaka D, Kapoor R, Bahl A, Bansal A, Mittal BR, et al. (2015) Impact of Integrating Pet-Ct in Radiotherapy Planning of Non-small Cell Carcinoma Lung: Dosimetric and Radiobiological Comparison. J Integr Oncol 4: 139. doi:10.4172/2329-6771.1000139

Page 5 of 6

statistically significant less maximum dose to the esophagus and also lesser volume of esophagus receiving doses of 50-55Gy. Similar results were seen in a study by Van de Wel et al. which showed a statistically significant decrease in the V55 of esophagus by about $8.7 \%$ [19]. In this study even the decrease in the maximum dose to esophagus reached statistical significance. De Ruysscher et al. has also shown a decrease in V55 by $10.4 \%$ which was statistically significant [12].

In this study we have also evaluated the impact of PETCT on the maximum dose to the spinal cord. About $3.5 \mathrm{~Gy}$ less maximum dose was delivered to the spinal cord by using PETCT data in planning. Mah et al. has also shown a decrease in the spinal cord dose Dmax with use of PETCT in their study [18].

Impact of PETCT on dose to heart was analyzed and was found to have no statistical significant difference. But use of PETCT resulted in a decrease in the mean dose to the heart by $8.09 \%$. Mah et al. similarly did not demonstrate any significant difference in the mean dose or D33 to the heart in their study [18].

As discussed above PETCT resulted in a decrease in the dose to various organs at risk. When this decrease in dose was assessed using the LKB model to predict the NTCP, it was seen that PETCT resulted in a statistically significant decrease in the normal lung and spinal cord NTCP while the decrease in the NTCP for esophagus did not reach statistical significance.

In this study, the TCP calculations were only estimations. Though no dose escalation effect was studied by using PETCT as was done in other studies by De Ruysscher et al. and by van Der Wel et al., the TCP values for PETCT based tumor were statistically higher than CT based tumor volumes which can be explained by the better tumor coverage achieved by using PETCT for tumor delineation $[12,19]$.

Although incorporation of PETCT invaluably contributed in staging and RT planning and has significantly decreased the target volumes, thus leading to decreased dose to the critical structures as seen in this study, there are several other issues that remained unresolved. In this study no pathological verification of the FDG positive nodes was done. Secondly, on PETCT it is difficult to establish the margins between the tumor and normal tissue. The optimal PET volume and margins need to be standardized for RT planning and for proper comparison of literature data. Thirdly, there is no consensus regarding the margins around the GTV that are necessary if PETCT data are used. In this study similar margins were given both for GTV/MTV contoured on CT and PETCT. Fourthly, we did not use respiratory correlated CT scan or 4D PETCT, so variation in degree of tumor motion existed. Similarly due to respiratory motion chances of mediastinal lymph node localization were also blurred.

\section{Conclusion}

Incorporation of PETCT into radiotherapy planning of NSCLC described herein provides a technically feasible and dosimetrically appealing strategy for treatment of patients. Use of PETCT resulted in better delineation of primary tumor differentiating it from atelectasis and also better identification of the involved lymph nodes. The resulting target volumes delineated thus decreased and this decrease in the target volume resulted in less radiation dose to various normal tissues. This was appreciated by the decrease in the NTCP values as was demonstrated with the use of PETCT.

Thus PETCT based radiotherapy planning can be considered the modality of choice for NSCLC but a well matched clinical correlation is required to find out local tumor control, various normal tissue complications, patterns of failure and long term toxicity.

\section{Ethics Approval and Compliance with Ethical Standards}

All procedures performed in our study involving human participants were in accordance with the ethical standards of the institutional and/or national research committee and with the 1964 Helsinki declaration and its later amendments or comparable ethical standards".

\section{References}

1. Qiao X, Tullgren O, Lax I, Sirzén F, Lewensohn R (2003) The role of radiotherapy in treatment of stage I non-small cell lung cancer. See comment in PubMed Commons below Lung Cancer 41: 1-11.

2. Kalff V, Hicks RJ, MacManus MP, Binns DS, McKenzie AF, et al. (2001) Clinical impact of (18)F fluorodeoxyglucose positron emission tomography in patients with non-small-cell lung cancer: a prospective study. Journal of clinical oncology 19: 111-118.

3. Vanuytsel LJ, Vansteenkiste JF, Stroobants SG, De Leyn PR, De Wever W, et al. (2000) The impact of (18)F-fluoro-2-deoxy-D-glucose positron emission tomography (FDG-PET) lymph node staging on the radiation treatment volumes in patients with non-small cell lung cancer. Radiother Oncol 55: 317-324.

4. De Ruysscher D, Nestle U, Jeraj R, Macmanus M (2012) PET scans in radiotherapy planning of lung cancer. See comment in PubMed Commons below Lung Cancer 75: 141-145.

5. Emami B, Lyman J, Brown A, Coia L, Goitein M, et al. (1991) Tolerance of normal tissue to therapeutic irradiation. See comment in PubMed Commons below Int J Radiat Oncol Biol Phys 21: 109-122.

6. Lyman JT (1985) Complication probability as assessed from dose volume histograms. Radiat Res 8: S13-S19.

7. Burman C, Kutcher GJ, Emami B, Goitein M (1991) Fitting of normal tissue tolerance data to an analytic function. See comment in PubMed Commons below Int J Radiat Oncol Biol Phys 21: 123-135.

8. Kutcher GJ, Burman C, Brewster L, Goitein M, Mohan R (1991) Histogram reduction method for calculating complication probabilities for three-dimensional treatment planning evaluations. Int J Radiat Biol Oncol Phys 21: 137-146.

9. Jameson MG, Kumar S, Vinod SK, Metcalfe PE, Holloway LC (2014) Correlation of contouring variation with modeled outcome for conformal non-small cell lung cancer radiotherapy. Radiother Oncol 112: 332-336.

10. Lebioda A, Makarewicz R, Malkowski B, Dancewicz M, Kowalewski J, et al. (2013) Measurement of primary tumor volume by PET-CT to evaluate risk of mediastinal nodal involvement in NSCLC patients with clinically negative N2 lymph nodes. Rep Pract Oncol Radiother 18: 76-81.

11. Bradley J, Thorstad WL, Mutic S, Miller TR, Dehdashti F, et al. (2004) Impact of FDG-PET on radiation therapy volume delineation in non-small-cell lung cancer. See comment in PubMed Commons below Int J Radiat Oncol Biol Phys 59: 78-86

12. De Ruysscher D, Wanders S, Minken A, Lumens A, Schiffelers J, et al. (2005) Effects of radiotherapy planning with a dedicated combined PET-CT-simulator of patients with non-small cell lung cancer on dose limiting normal tissues and radiation dose-escalation: a planning study. Radiother Oncol 77: 5-10.

13. Deniaud-Alexandre E, Touboul E, Lerouge D, Grahek D, Foulquier JN, et al. (2005) Impact of computed tomography and 18F-deoxyglucose coincidence detection emission tomography image fusion for optimization of conforma radiotherapy in non-small-cell lung cancer 63: 1432-1441.

14. Ashamalla H, Rafla S, Parikh K, Mokhtar B, Goswami G, et al. (2005) The contribution of integrated $\mathrm{PET} / \mathrm{CT}$ to the evolving definition of treatment volumes in radiation treatment planning in lung cancer. See comment in PubMed Commons below Int J Radiat Oncol Biol Phys 63: 1016-1023.

15. Erdi YE, Rosenzweig K, Erdi AK, Macapinlac HA, Hu YC, et al. (2002) Radiotherapy treatment planning for patients with non-small cell lung cancer using positron emission tomography (PET). See comment in PubMed Commons below Radiother Oncol 62: 51-60.

16. Brianzoni E, Rossi G, Ancidei S, Berbellini A, Capoccetti F, et al. (2005) Radiotherapy planning: PET/CT scanner performances in the definition of 
Citation: Koppaka D, Kapoor R, Bahl A, Bansal A, Mittal BR, et al. (2015) Impact of Integrating Pet-Ct in Radiotherapy Planning of Non-small Cell Carcinoma Lung: Dosimetric and Radiobiological Comparison. J Integr Oncol 4: 139. doi:10.4172/2329-6771.1000139

gross tumour volume and clinical target volume. Eur $\mathrm{J}$ Nucl Med Mol Imaging 32: $1392-1399$

17. Schmucking M, Baum RP, Griesinger F, Presselt N, Bonnet R, et al. (2003) Molecular whole-body cancer staging using positron emission tomography: consequences for therapeutic management and metabolic radiation treatment planning. Recent Results Cancer 162: 195-202.

18. Mah K, Caldwell CB, Ung YC, Danjoux CE, Balogh JM, et al. (2002) The impact of (18)FDG-PET on target and critical organs in CT-based treatment planning of patients with poorly defined non-small-cell lung carcinoma: a prospective study. Int J Radiat Oncol Biol Phys 52: 339-350.

19. Van Der Wel A, Nijsten S, Hochstenbag M, Lamers R, Boersma L, et al. (2005) Increased therapeutic ratio by 18 FDG-PET CT planning in patients with clinical CT stage N2-N3M0 non-small-cell lung cancer: a modeling study. Int J Radiat Oncol Biol Phys 61: 649-655 